\section{ЭКСПЕРИМЕНТАЛЬНОЕ МОДЕЛИРОВАНИЕ ФОРМИРОВАНИЯ ТРЕЩИН ОТРЫВА У КРУГЛОЙ ВЫРАБОТКИ}

\author{
С. В. Сукнев
}

Институт горного дела Севера им. Н. В. Черского СО РАН, E-mail: suknyov@igds.ysn.ru, просn. Ленина 43, г. Якутск 677980, Россия

\begin{abstract}
Экспериментально исследовано формирование трещин отрыва в геосреде, содержащей цилиндрическую полость и подверженную неравномерно распределенному сжатию. Обнаружено существенное влияние диаметра отверстия на локальную прочность материала. Предложен новый критерий квазихрупкого разрушения материала в зоне концентрации растягивающих напряжений, содержащий комплексный параметр, характеризующий размер зоны предразрушения и учитывающий не только структуру материала, но и его пластические свойства, геометрию выработки и условия нагружения. Результаты расчетов хорошо согласуются с полученными экспериментальными данными.
\end{abstract}

Сжатие, геосреда, иилиндрическая полость, квазихрупкое разрушение, масштабньй эффект, градиентный критерий

\title{
EXPERIMENTAL SIMULATION OF TENSILE FRACTURING INITIATION FROM A CIRCULAR CAVITY
}

\author{
S. V.Suknev \\ Chersky Institute of Mining of the North, Siberian Branch, Russian Academy of Sciences, \\ E-mail: suknyov@igds.ysn.ru, pr. Lenina 43, Yakutsk 677980, Russia
}

\begin{abstract}
The paper presents results of the experimental studies on tensile fractures formation in the geomedium with a cylindrical cavity and subjected to non-uniformly distributed compression. The hole diameter was found to have a significant effect on the local strength of the material. The new criterion proposed for quasi-brittle fracturing contains a complex parameter that characterizes the size of the pre-fracture zone and accounts not only for the material's microstructure, but also for its plastic properties, as well as for geometry of the cavity, and loading conditions. Calculation results are in good agreement with the experimental data.
\end{abstract}

Compression, geomedium, cylindrical cavity, quasi-brittle fracture, size effect, stress gradient criterion

Горные породы характеризуются низкой прочностью на растяжение по сравнению с прочностью на сжатие. При этом даже в условиях действия сжимающих нагрузок возле горных выработок могут возникать зоны растягивающих напряжений и образовываться трещины отрыва (разрывы породы), что представляет серьезную опасность.

Кроме того, структурно-неоднородные материалы, в том числе горные породы, демонстрируют зависимость прочностных свойств от нагруженного объема (масштабный эффект), наиболее сильно проявляющуюся в условиях концентрации напряжений, когда эффективный нагруженный объем определяется зоной концентрации напряжений, размер которой мал по сравне-

Работа выполнена при финансовой поддержке Российского фонда фундаментальных исследований (проект № 18-05-00323). 
нию с характерными размерами деформируемого тела. В этих случаях для расчета критической нагрузки используют нелокальные и градиентные критерии разрушения [1-11]. Нелокальные критерии основаны на представлении о формировании в материале зоны предразрушения, в которой происходит локальное перераспределение напряжений, в то время как основной материал деформируется упруго вплоть до разрушения. Альтернативная точка зрения на масштабный эффект состоит в том, что в хрупких материалах он связан не с перераспределением напряжений, а с реальным увеличением локального разрушающего напряжения. Концепция локальной прочности лежит в основе градиентного критерия разрушения [12].

В данной работе экспериментально и теоретически исследовано влияние диаметра кругового отверстия на разрушение структурно-неоднородного геоматериала в зоне концентрации напряжений при неравномерно распределенном сжатии с учетом масштабного эффекта и выполнен анализ возможности применения градиентного критерия для описания квазихрупкого разрушения материала.

\section{МЕТОДИКА ЭКСПЕРИМЕНТА}

Испытывали гипсовые образцы с круговым отверстием под действием неравномерно распределенной сжимающей нагрузки. Образцы изготавливали из водного раствора строительного гипса, приготовленного в соотношении (по весу) 1 часть воды на 1.5 части гипса. Образцы представляли собой квадратные плиты размером 200×200 мм и толщиной 36 мм. После изготовления их высушивали на воздухе в течение 30-40 сут, плотность образцов в сухом состоянии составила 1.10 г/см³. Перед испытанием в центре образцов высверливали круговые отверстия различного диаметра от 1 до 20 мм. Нагрузку $p$ прикладывали к образцу через жесткие вставки размером 120 мм, помещенные между образцом и нагружающими плитами испытательной машины (рис. 1).

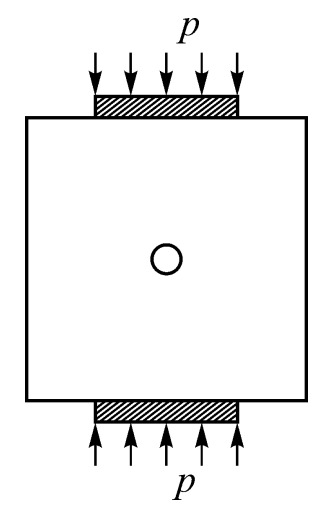

Рис. 1. Схема нагружения образца

Было исследовано по 5 образцов с отверстиями каждого диаметра. В процессе испытания в зонах концентрации растягивающих напряжений на контуре отверстия наблюдали образование трещин отрыва, которое носило внезапный характер. В образцах с отверстием диаметром от 5 до 20 мм трещины быстро распространялись на расстояние около 50 мм вдоль линии приложения сжимающей нагрузки, при дальнейшем нагружении их рост стабилизировался. В образцах с отверстием диаметром 1 и 2 мм трещины моментально распространялись практически на все вертикальное сечение образца. Образование трещин также сопровождалось локальной разгрузкой образца, что отражалось на диаграмме деформирования в виде появления зубца. Наибольшей разгрузке оказались подвержены образцы с отверстием наименьшего диаметра 1 мм. На рис. 2 показаны характерные диаграммы деформирования образцов с отверстиями различного диаметра в виде скриншота окна программы testXpert с результатами испытаний. Критическую нагрузку в момент образования трещин определяли по вершине зубца на диаграмме. 


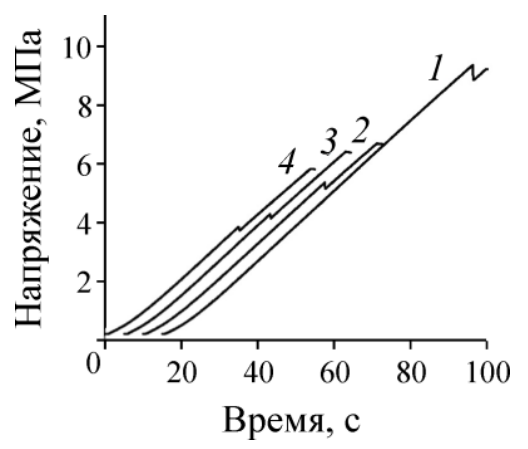

Рис. 2. Диаграммы деформирования образцов с отверстиями различного диаметра, мм: $1-1$; $2-5 ; 3-10 ; 4-15$

Для определения прочности материала на сжатие использовали такие же квадратные образцы размером $200 \times 200$ мм без отверстия. Нагружение проводили через вставки размером 200 мм, значение предела прочности составило 11.53 МПа. Предел прочности материала на растяжение устанавливали на образцах корсетного типа с радиусом закругления рабочей части 110 - 120 мм и шириной в минимальном сечении 29 мм, значение предела прочности $2.61 \mathrm{MПа.}$

\section{ТЕОРЕТИЧЕСКИЙ ПОДХОД}

В соответствии с градиентным критерием [12] локальная прочность материала предполагается зависимой от размера зоны концентрации напряжений $L_{e}$. Если размер $L_{e}$ достаточно велик по сравнению с размерами структурных составляющих материала, то величина локальной прочности мало отличается от величины предельного напряжения $\sigma_{0}$, определенной в условиях однородного распределения напряжений. Наоборот, если $L_{e}$ сопоставим с размерами структурных элементов, их влияние на локальную прочность становится заметным. Причем это влияние тем больше, чем меньше размер $L_{e}$ по отношению к характерному размеру структуры материала $L_{0}$. Таким образом, локальная прочность материала зависит не просто от размера зоны концентрации напряжений $L_{e}$, а от соотношения $L_{0} / L_{e}$, которое характеризует масштаб в рассматриваемой задаче. С учетом этого критерий представляется в виде $\sigma_{e}<f\left(\sigma_{0}, L_{0} / L_{e}\right)$, где $\sigma_{e}$ - эквивалентное напряжение, характеризующее внутреннее напряженное состояние деформируемого тела. Для задачи об образовании трещин отрыва при сжатии эквивалентное напряжение определяется по теории наибольших растягивающих напряжений, а функция локальной прочности имеет вид:

$$
f\left(\sigma_{0}, L_{0} / L_{e}\right)=\sigma_{0}\left(1+\left(\frac{L_{0}}{L_{e}}\right)^{n}\right)
$$

где $\sigma_{0}$ - предел прочности материала при растяжении; $L_{e}=\frac{\sigma_{e}}{\left|\operatorname{grad} \sigma_{e}\right|}$. Критическое напряжение, при котором на контуре отверстия образуются трещины отрыва, определяется выражением

$$
\sigma_{c}=\frac{\sigma_{0}}{k}\left(1+\left(\frac{L_{0}}{L_{e}}\right)^{n}\right) .
$$

Коэффициент $k$ в формуле (2) представляет собой отношение максимального растягивающего напряжения на контуре отверстия к величине приложенного напряжения $\sigma$. Для хрупких материалов показатель степени $n=1$. При значении параметра $L_{0}=0$ формула (2) дает расчет критического напряжения согласно традиционному критерию разрушения. 
В результате нагружения образца по схеме, приведенной на рис. 1, в центральной части (вне зоны влияния отверстия) реализуется достаточно однородное двухосное напряженное состояние: растяжение усилиями $\alpha \sigma$ по горизонтальной оси и сжатие усилиями $\sigma$ по вертикальной оси образца (рис. 3).

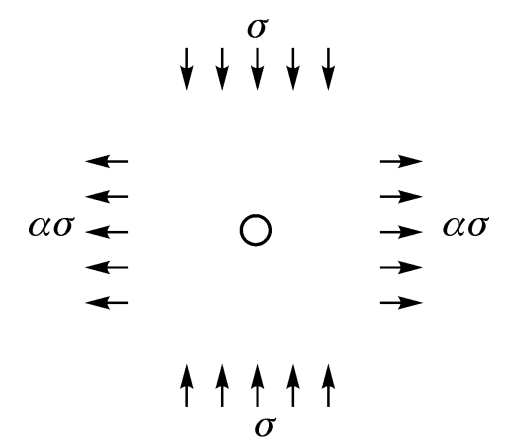

Рис. 3. Круговое отверстие при двухосном нагружении

Значения $\sigma$ и $\alpha$ рассчитывали методом конечных элементов в центре образцов, нагруженных через вставки заданного размера и не содержащих отверстия. Для использованных в экспериментах вставок $\sigma=0.764 p, \alpha=0.187$.

В соответствии с известным решением задачи Кирша распределение нормального напряжения $\sigma_{y}$ вдоль линии приложения сжимающей нагрузки имеет вид:

$$
\sigma_{y}=\frac{\sigma}{2}\left(3 \frac{a^{4}}{x^{4}}-\frac{a^{2}}{x^{2}}\right)+\frac{\alpha \sigma}{2}\left(2+\frac{a^{2}}{x^{2}}+3 \frac{a^{4}}{x^{4}}\right),
$$

где $a$ - радиус отверстия. Начало координат выбрано в центре отверстия, величина приложенного сжимающего напряжения $\sigma$ принята положительной. Таким образом, с учетом выражения (3) для рассматриваемой задачи получим: $k=1+3 \alpha, L_{e}=a \frac{1+3 \alpha}{5+7 \alpha}$.

Градиентный критерий в исходной формулировке не предполагает образования в материале зоны поврежденности, поэтому его применение, строго говоря, ограничено разрушением хрупких материалов. Для описания квазихрупкого разрушения, следуя [13], представим структурный параметр $L_{0}$ в следующем виде:

$$
L_{0}=d_{0}+\beta L_{e},
$$

где $d_{0}$ - размер структуры материала; $\beta$ - безразмерный параметр, характеризующий пластичность материала. Для хрупких материалов $\beta=0$, для пластичных $-\beta>1$. При $\beta \sim 1$ материал обладает умеренными пластическими свойствами. Первое слагаемое в выражении (4) описывает собственно структуру материала, а второе отражает вклад неупругих деформаций.

В первоначальной формулировке критерия (при $\beta=0$ ) параметр $L_{0}$ имел смысл размера структуры материала $d_{0}$ и не ассоциировался с зоной предразрушения. С учетом выражения (4) $L_{0}$ приобретает смысл параметра $d$ (размер зоны предразрушения) в нелокальных критериях [13], а увеличение локальной прочности (1) связано не только с реальным масштабным эффектом, характерным для хрупких материалов, но также с “видимым” (кажущимся) увеличением за счет перераспределения упругих напряжений в зоне предразрушения.

В соответствии с формулой (2) и с учетом сделанных оценок для $\sigma$ и $\alpha$ запишем выражение для критического давления в образце с круговым отверстием:

$$
p_{c}=0.838 \chi C_{0}\left(1+8.081 \frac{d_{0}}{l}+\beta\right),
$$


где $\chi=\sigma_{0} / C_{0} ; C_{0}$ - предел прочности материала при сжатии; $l$ - диаметр отверстия. Первое слагаемое в выражении (5) отвечает расчету разрушающей нагрузки согласно традиционному подходу, не учитывающему масштабный эффект, второе слагаемое определяет вклад в масштабный эффект реального увеличения локальной прочности материала, а третье “видимого". Асимптотическое при $l \rightarrow \infty$ значение критического давления $T_{s}=T_{0}(1+\beta)$, где $T_{0}=0.838 \chi C_{0}-$ асимптотическое значение критического давления для хрупкого материала.

\section{РЕЗУЛЬТАТЫ И ОБСУЖДЕНИЕ}

На рис. 4 представлены экспериментальные данные (точки) о величине нагрузки в момент образования трещин отрыва на контуре отверстия в зависимости от его диаметра и результаты расчета критического давления по формуле (5) при значениях $\beta=0$ (кривая 1 ) и $\beta=0.3$ (кривая 2). Размер $d_{0}$ составил 0.5 мм. Асимптотическое значение критического давления в первом случае равно $T_{0}$ (штриховая прямая), во втором $-T_{s}=1.3 T_{0}$ (сплошная прямая).

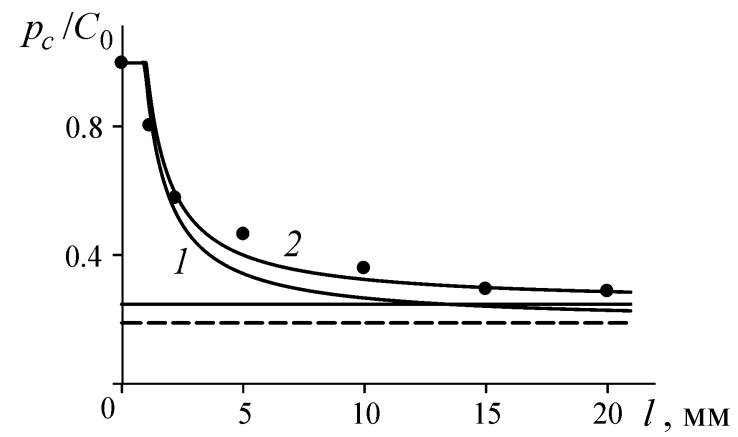

Рис. 4. Зависимость критического давления от диаметра отверстия

Рис. 4 иллюстрирует существенный масштабный эффект, т. е. влияние диаметра отверстия на локальную прочность материала. С его уменьшением критическое давление возрастает, достигая предела прочности на сжатие, с увеличением - асимптотически приближается к напряжению $T_{s}$.

Применение исходного градиентного критерия разрушения (при $\beta=0$ ) для описания экспериментальных данных позволяет получить удовлетворительные оценки величины критического давления только при малых (1-2 мм) диаметрах отверстия. Результаты расчетов, выполненных для больших диаметров отверстия, дают заниженные значения критического давления. Экспериментальные данные свидетельствуют о том, что при увеличении диаметра отверстия критическое давление асимптотически стремится к значению, превышающему на 30 \% значение, рассчитанное для упругого тела. При этом разрушение испытанных образцов сопровождается внезапным образованием на контуре отверстия и быстрым распространением вдоль оси сжатия трещин отрыва. Все это позволяет охарактеризовать разрушение данного материала в исследованном диапазоне диаметров отверстия как квазихрупкое. Такое поведение хорошо описывается модифицированным градиентным критерием разрушения, в котором параметр $L_{0}$ определяется по формуле (4).

\section{ВЫводы}

Теоретически и экспериментально исследовано разрушение модельного геоматериала, содержащего круговое отверстие, при неравномерно распределенном сжатии и осуществлен анализ возможности применения градиентного критерия для оценки разрушающей нагрузки. Испытанные образцы продемонстрировали квазихрупкий характер разрушения. В этом случае применение обычного градиентного критерия не позволяет получить удовлетворительные оценки разрушающей нагрузки. Поэтому для расчета разрушающей нагрузки предложено ис- 
пользовать модифицированный градиентный критерий, содержащий комплексный параметр, характеризующий размер зоны предразрушения и учитывающий не только структуру материала, но также пластические свойства материала, геометрию образца и условия его нагружения. Результаты расчетов по модифицированному критерию хорошо согласуются с полученными экспериментальными данными.

\section{СПИСОК ЛИТЕРАТУРЫ / REFERENCES}

1. Novozhilov V. V. On a necessary and sufficient criterion for brittle strength, J. Appl. Math. Mech., 1969, vol. 33, no. 2, pp. 201-210 [Новожилов В. В. О необходимом и достаточном критерии хрупкой прочности // Прикл. математика и механика. — 1969. — Т. 33. — № 2. — С. 212 -222.]

2. Lajtai E. Z. Effect of tensile stress gradient on brittle fracture initiation, Int. J. Rock. Mech. Min. Sci., 1972, vol. 9, no. 5, pp. 569-578.

3. Whitney J. M. and Nuismer R. J. Stress fracture criteria for laminated composites containing stress concentrations, J. Compos. Mater., 1974, vol. 8, no. 4, pp. 253-265.

4. Carter B. J., Lajtai E. Z., and Yuan Y. Tensile fracture from circular cavities loaded in compression, Int. J. Fract., 1992, vol. 57, no. 3, pp. 221-236.

5. Seweryn A. and Mroz Z. A non-local stress failure condition for structural elements under multiaxial loading, Eng. Fract. Mech., 1995, vol. 51, no. 6, pp. 955-973.

6. Mikhailov S. E. A functional approach to non-local strength condition and fracture criteria, Eng. Fract. Mech., 1995, vol. 52, no. 4, pp. 731-754.

7. Suknev S. V. and Novopashin M. D. Determination of local mechanical properties of materials, Proceedings of the Academy of Sciences, 2000, vol. 45, no. 7, pp. 339-341 [Сукнев С. В., Новопашин М. Д. Определение локальных механических свойств материалов // ДАН. - 2000. — Т. 373. — № 1. - С. 48 -50.]

8. Cornetti P., Pugno N., Carpinteri A., and Taylor D. Finite fracture mechanics: a coupled stress and energy failure criterion, Eng. Fract. Mech., 2006, vol. 73, no. 14, pp. $2021-2033$.

9. Taylor D. The theory of critical distances: a new perspective in fracture mechanics. Oxford: Elsevier, 2007.

10. Torabi A. R. and Pirhadi E. Stress-based criteria for brittle fracture in key-hole notches under mixed mode loading, Eur. J. Mech. A/Solids, 2015, vol. 49, pp. 1-12.

11. Sapora A., Torabi A. R., Etesam S., Cornetti P. Finite Fracture Mechanics crack initiation from a circular hole, Fatigue Fract. Eng. Mater. Struct., 2018, vol. 41, no. 7, pp. 1627-1636.

12. Suknev S. V. and Novopashin M. D. Criterion of normal tension crack formation in rocks under compression, Journal of Mining Science, 2003, vol. 39, no. 2, pp. 132 - 138 [Сукнев С. В., Новопашин М. Д. Критерий образования трещин отрыва в горных породах при сжатии // ФТПРПИ. - 2003. — № 2. C. $30-37$.

13. Suknev S. V. Nonlocal and gradient fracture criteria for quasi-brittle materials under compression, Physical Mesomechanics, 2018, vol. 21, no. 4, pp. 22 -32 (in Russian) [Сукнев С. В. Нелокальные и градиентные критерии разрушения квазихрупких материалов при сжатии // Физическая мезомеханика. 2018. - T. 21. — № 4. - C. 22-32.] 